\title{
Isotope-Selective Detection and Imaging of Organic Nanolayers
}

2009

Vol. 9, No. 8 3020-3024

\author{
H. J. Mamin, ${ }^{*, \dagger}$ T. H. Oosterkamp, ${ }^{\ddagger}$ M. Poggio, ${ }^{, \S, \|}$ C. L. Degen, ${ }^{t, \ddagger}$ C. T. Rettner, ${ }^{\dagger}$ \\ and D. Rugar ${ }^{\dagger}$
}

IBM Research Division, Almaden Research Center, 650 Harry Road, San Jose, California 95120, Leiden Institute of Physics, Leiden University, Leiden, The Netherlands, and Center For Probing the Nanoscale, Stanford University, 476 Lomita Mall, Stanford, California 94305

Received May 7, 2009

\begin{abstract}
Magnetic resonance force microscopy (MRFM) makes use of the spectroscopic nature of magnetic resonance to add unambiguous elemental selectivity to scanning probe microscopy. We show isotopic selectivity of MRFM for three nuclei, ${ }^{1} \mathrm{H},{ }^{31} \mathrm{P}$, and ${ }^{13} \mathrm{C}$, in organic materials. We also detect a roughly $1 \mathrm{~nm}$ thick layer of naturally occurring adsorbates on a gold surface by measuring the magnetic resonance signal of the hydrogen contained in the layer. Finally, we detect the signal from hydrogen present on a carbon nanotube and use it to perform a threedimensional magnetic resonance image of the $10 \mathrm{~nm}$ diameter object.
\end{abstract}

Adding chemical contrast to the topographic capabilities of scanning probe microscopy is a longstanding aspiration, dating back to the early proposals by Binnig and Rohrer to monitor variations in the local tunneling barrier with the scanning tunneling microscope (STM). ${ }^{1}$ This goal has led to great interest in combining scanning probes with spectroscopic techniques such as inelastic tunneling spectroscopy, ${ }^{2}$ force spectroscopy, ${ }^{3}$ and Raman spectroscopy, ${ }^{4}$ all of which have successfully demonstrated atomic or molecular identification in certain systems. In general, however, scanning probe microscopes are not particularly well-equipped to identify what they are imaging. Magnetic resonance imaging (MRI), on the other hand, is an imaging technique that is inherently selective to specific elements based on nuclear magnetic resonance (NMR). We have recently demonstrated that the technique of magnetic resonance force microscopy (MRFM) ${ }^{5,6}$ can give a 100-million fold improvement in the volume resolution compared to MRI by using ultrasensitive cantilever-based detection, thus providing a nanoscale imaging technique that is intrinsically elementspecific. ${ }^{7}$ Here we demonstrate this selectivity in organic materials by detecting three nuclear species, ${ }^{1} \mathrm{H},{ }^{31} \mathrm{P}$, and ${ }^{13} \mathrm{C}$. We further use the technique's sensitivity to hydrogen to detect the naturally occurring layer of adsorbates that builds

* To whom correspondence should be addressed. E-mail: mamin@ almaden.ibm.com. Phone: 408-927-2502.

IBM Research Division.

* Leiden University.

$\S$ Stanford University.

"Current address: Department of Physics, University of Basel, Klingelbergstrasse $82, \mathrm{CH}-4056$ Basel.

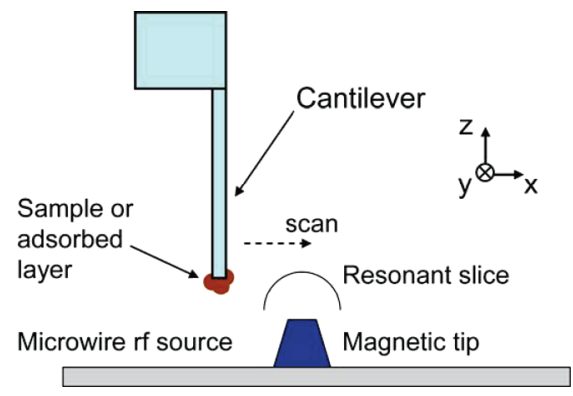

Figure 1. Experimental setup for magnetic resonance force microscopy (MRFM) with sample-on-cantilever geometry. The sample in these experiments is either a particle of organic material, a hydrocarbon/water layer adsorbed on the gold-coated end of the silicon cantilever, or a carbon nanotube protruding from the cantilever.

up on a cleaned gold surface. Finally, we use the hydrogencontaining adsorbate layer as a "contrast agent" to perform three-dimensional imaging of a multiwalled carbon nanotube.

In MRFM, the sample is scanned relative to a magnetic tip while the sample's nuclear magnetism is manipulated through an applied rf field. The sample's net magnetic moment is then sensed through ultrasensitive force detection. ${ }^{5,6,8}$ As shown in the setup in Figure 1, a magnetic tip supplies an inhomogenous static field $\mathbf{B}_{\text {tip }}(\mathbf{r})$. A rf current flowing through a wire in the $y$-direction generates a rf field at angular frequency $\omega_{\mathrm{rf}}$ that points along the $x$ direction directly above the tip. ${ }^{9}$ Typical rf frequencies are in the range of 10-200 MHz. Magnetic resonance can occur when the total field at a given location $\mathbf{r}$ satisfies the condition $B_{\mathrm{tot}} \equiv$ 


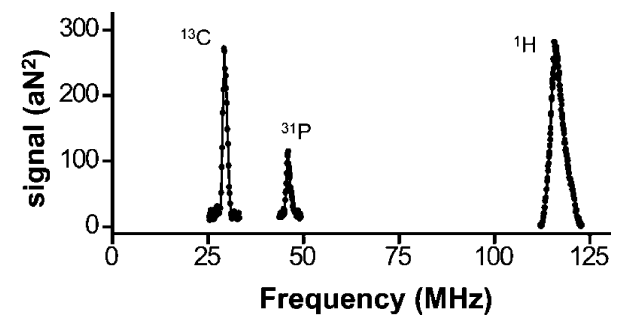

Figure 2. MRFM spectroscopy showing signal peaks at different frequencies of the applied rf field, corresponding to the specific gyromagnetic ratios of the nuclear spins. The peaks at 29 and 116 $\mathrm{MHz}$ correspond to ${ }^{13} \mathrm{C}$ and ${ }^{1} \mathrm{H}$, respectively, and were obtained using a particle of ${ }^{13} \mathrm{C}$-enriched stearic acid attached to the end of the cantilever. The peak at $46 \mathrm{MHz}$ was obtained on a particle of lyophilized DNA attached to the cantilever and corresponds to ${ }^{31} \mathrm{P}$. A systematic offset of $60 \mathrm{aN}^{2}$ has been subtracted from the latter curve. The ${ }^{31} \mathrm{P}$ curve was obtained at $280 \mathrm{mK}$ with an external applied field of $2.61 \mathrm{~T}$, while the other curves were taken at $4.2 \mathrm{~K}$ at $2.64 \mathrm{~T}$. Because the data sets for each isotope were taken under slightly different experimental conditions, the relative signal amplitudes are somewhat arbitrary.

$\left|\mathbf{B}_{\text {ext }}+\mathbf{B}_{\text {tip }}(\mathbf{r})\right|=\omega_{\text {rf }} / \gamma$. Here $\mathbf{B}_{\text {ext }}$ is a static field applied in the $z$-direction, and $\gamma$ is the gyromagnetic ratio. The gyromagnetic ratio depends on the magnetic moment of the nuclear spin, which differs for different elements. For the elements used here, $\gamma^{1}{ }_{\mathrm{H}} / 2 \pi=42.58 \mathrm{MHz} / \mathrm{T}, \gamma^{31 \mathrm{p}} / 2 \pi=17.24$ $\mathrm{MHz} / \mathrm{T}$, and $\gamma^{13} \mathrm{C} / 2 \pi=10.71 \mathrm{MHz} / \mathrm{T}$. Because of the large field gradient from the magnetic tip, the resonance condition is satisfied only within a thin, bowl-shaped "resonant slice".

To generate an oscillatory force signal, the frequency of the rf field is swept in a sawtooth pattern about the center frequency $\gamma B_{\text {tot }}$, thus driving adiabatic inversions of the spins of interest at the cantilever resonant frequency. The spins then generate an oscillating force on the cantilever, resulting in vibration of the cantilever of order $0.1-1 \AA$. The microwire if source, ${ }^{9}$ magnetic tip, and measurement protocol used here are identical to those used in a previous MRFM nanoscale imaging experiment. ${ }^{7}$ In particular, we detect the statistical polarization that originates from the naturally occurring statistical fluctuations in the net polarization. These fluctuations give rise to a time-varying signal with zero mean and a variance proportional to $N$, the number of contributing spins. ${ }^{10-13}$ As the sample is scanned through the resonant slice, the signal will depend on how much of the object intersects the resonant slice. By knowing the shape of the resonant slice (i.e., the point spread function of the microscope), a three-dimensional (3D) scan of signal versus position can be deconvolved to determine a real-space 3D image of the sample. ${ }^{7,14,15}$ This capability for resolving subsurface structures is a key feature of MRFM and distinguishes the method from conventional scanning probe microscopies that are typically restricted to surfaces.

Figure 2 reveals the elemental selectivity of the technique. The graph combines data taken from two experiments in which particles of organic material were attached onto the ends of custom mass-loaded cantilevers of the type described below. In one experiment, a roughly $(5 \mu \mathrm{m})^{3}$ particle of ${ }^{13} \mathrm{C}$ labeled stearic acid was used, and in the other, a similarly sized particle of lyophilized DNA. At given external fields and tip positions, signals were observed at specific rf frequencies that can be associated with particular elements using the known gyromagnetic ratios. In this case, we observed the ${ }^{1} \mathrm{H}$ and the isotopically enriched ${ }^{13} \mathrm{C}$ in the stearic acid sample, and ${ }^{31} \mathrm{P}$ in the DNA sample. $\left({ }^{1} \mathrm{H}\right.$ was also detected in the DNA sample). We note that DNA contains only one phosphorus atom per nucleotide, resulting in a density roughly 10 times lower density than that of hydrogen. While such spectroscopic capabilities have previously been demonstrated in MRFM measurements of III/V semiconductors and other inorganic systems, ${ }^{16,17}$ here we demonstrate elemental selectivity in organic materials.

One system of particular interest to us is that of naturally occurring adsorbate layers on nominally clean gold surfaces. We are motivated in this work not only by the fact that hydrocarbon and water adsorption are ubiquitous phenomena that can dramatically affect surface chemistry, ${ }^{18,19}$ but also because of some peculiar results in previous MRFM imaging. We recently performed three-dimensional imaging of tobacco mosaic viruses (TMV) with resolution down to $4 \mathrm{~nm}$ by detecting the hydrogen nuclei in the viruses. ${ }^{7}$ However, the MRFM images also revealed an unexpected feature: the viruses sat on a layer of hydrogen-rich material of the order of $1 \mathrm{~nm}$ thick.

In order to address the source, we present here a control experiment designed to test whether the layer was intrinsic or related to the dipping of the cantilever into the viruscontaining buffer solution. The cantilever preparation was identical to that used in the TMV experiment, except for the final dipping step. The cantilever had a custom massloaded design, incorporating a roughly $1 \mu \mathrm{m}$ thick mass on the end of a $90 \mu \mathrm{m}$ long, $100 \mathrm{~nm}$ thick shaft. ${ }^{20}$ The cantilever spring constant was $8.6 \times 10^{-5} \mathrm{~N} / \mathrm{m}$ and its resonant frequency was $2800 \mathrm{~Hz}$. A focused ion beam was used to mill a flat end to the cantilever, which was then coated endon with $10 \mathrm{~nm} \mathrm{Si} / 30 \mathrm{~nm}$ Au via thermal evaporation. Next the cantilever was UV-ozone cleaned and exposed to ambient conditions for roughly $3 \mathrm{~h}$ before placing in vacuum. The sample was pumped in moderate vacuum $\left(<10^{-6}\right.$ torr $)$ for several days before cooling down to $280 \mathrm{mK}$.

A distinct magnetic resonance signal corresponding to hydrogen was observed, as shown in the plot of signal versus external field in Figure 3a. The signal is similar in magnitude to the background signal observed in the TMV experiment. This result shows that the background layer is an intrinsic effect, apparently due to hydrogen-containing adsorbates picked up on the freshly cleaned gold surface during its exposure to ambient conditions. It is well known that gold surfaces cleaned via UV-ozone exposure are initially hydrophilic but become hydrophobic on the time scale of hours, presumably due to the same contamination mechanism..$^{21,22}$

A scaled fit to the data using the same tip model as used in the TMV experiment is also plotted and gives excellent agreement with the data. The symmetric shape of the curve is a clue that the signal arises from a thin layer, as theory and experiment indicate that a thick sample will give rise to a much more asymmetric line shape as the resonant slice pushes into the extended sample. ${ }^{23}$ From the fitted curve, a 

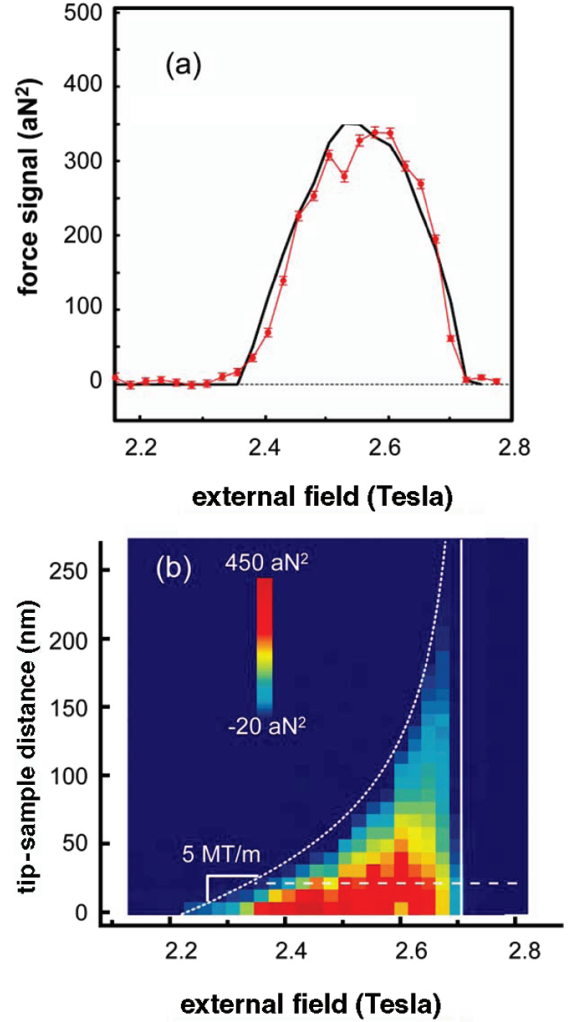

Figure 3. Magnetic resonance signal from a gold-coated control cantilever. The cantilever was cleaned via UV/ozone exposure, followed by a $3 \mathrm{~h}$ exposure to ambient conditions. (a) The signal as a function of external field shows the presence of a hydrogen signal. The points are the data, and the solid curve shows a theoretical fit that is used to extract parameters that describe the magnetic tip. The peak is inhomogenously broadened by the field gradient and the spatial extent of the sample. The frequency of the rf field was 114.7 MHz. (b) MRFM signal (false color) as a function of tip-sample spacing and external field. The data in (a) represent a horizontal cut through the region shown by the dashed line. The slope of the dotted line indicates a field gradient of $5 \times 10^{6} \mathrm{~T} / \mathrm{m}$.

quantitative value for the tip field at the given tip-sample spacing can be extracted, roughly $0.34 \mathrm{~T}$ in this case.

We can use the thin adsorbed hydrogen-containing layer as a test object in order to perform nanoscale quantitative magnetometry of our magnetic tip. The MRFM signal is shown as a function of external field and tip-sample spacing in the false color plot in Figure 3b. The distance between the solid line and the dotted line represents the tip field. The inverse slope of the dotted line therefore directly gives the tip field $z$-gradient $\partial B_{\text {tip }, z} / \partial z$. At external fields below $2.4 \mathrm{~T}$, corresponding to tip fields above $0.3 \mathrm{~T}$, the $z$-gradient is roughly $5 \times 10^{6} \mathrm{~T} / \mathrm{m}$. We can then use the measured $z$-gradient from the tip as an input parameter to determine an appropriate magnetic model for the tip and thereby calculate the point spread function (PSF) of the microscope.

On the basis of the measured force power of $350 \mathrm{aN}^{2}$ and the calculated PSF at the corresponding external field of 2.55 $\mathrm{T}$, we estimate that the resonant slice contains roughly $10^{6}$ spins. Assuming a hydrocarbon spin density of $7 \times 10^{28}$ spins per $\mathrm{m}^{3}$, the corresponding volume of spins is then $1.4 \times$
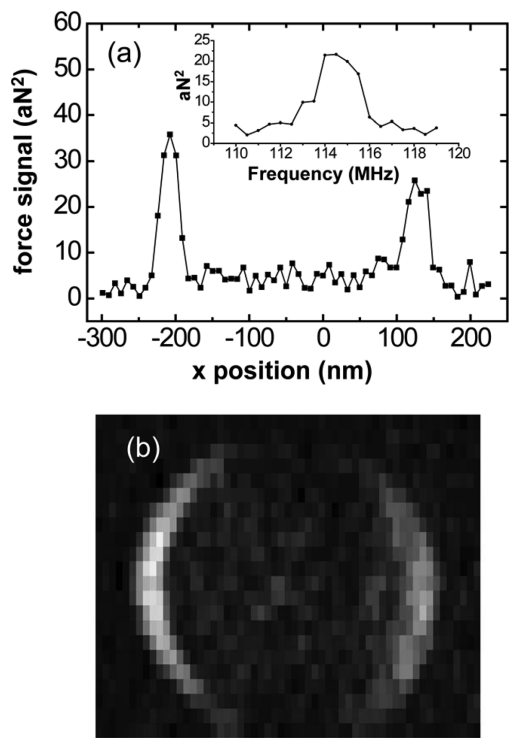

Figure 4. MRFM signal from a carbon nanotube. (a) Magnetic resonance signal vs lateral position over the magnetic tip. Signal is peaked near the edges of the tip. The frequency of the applied rf field was $115 \mathrm{MHz}$. Inset shows the signal at the tip edge vs frequency of the rf field. The data were acquired with a $1 \mathrm{~min}$ acquisition time per point. $B_{\text {ext }}=2.61 \mathrm{~T}$. $T=250 \mathrm{mK}$. (b) Two dimensional image of signal vs $x$ and $y$ position. The ring corresponds to a cut through the resonant slice and provides a visualization of the point spread function.

$10^{-23} \mathrm{~m}^{3}$. On the basis of the geometry of the PSF (diameter and width), we infer a layer thickness of roughly $10 \AA$.

We have also used the hydrogen signal as a form of contrast to allow imaging of a multiwalled nanotube (NT) in three dimensions. The NT was grown via the arc discharge method $^{24}$ and was roughly $10 \mathrm{~nm}$ in diameter. As shown in Figure 5a, the NT was attached to the end of a $100 \mathrm{~nm}$ thick cantilever so that it protruded a few hundred nanometers using a scanning electron microscope with an in situ custom micromanipulator. The cantilever had uniform thickness (no mass loading) with a spring constant of roughly $2 \times 10^{-4}$ $\mathrm{N} / \mathrm{m}$ and a resonant frequency of $9.1 \mathrm{kHz}$. The cantilever was stored under ambient conditions for several weeks before its placement in the apparatus.

While it was not obvious that the NT sample would show a hydrogen magnetic resonance signal, a clear signal was in fact observed when a rf field was applied near 115 $\mathrm{MHz}$, corresponding to the hydrogen resonance frequency $B_{\text {tot }} / \gamma^{1} \mathrm{H}$, as shown in Figure $4 \mathrm{a}$ (inset). The main plot in Figure 4a shows that the signal is peaked at the scan positions where the NT enters or exits the resonant slice. These are the positions where the NT has the most overlap with the resonant slice and where the lateral gradient is maximized. The peak signal is only about $35 \mathrm{aN}^{2}$, roughly $10 \times$ smaller than that from the adsorbed layer on the gold-coated cantilever, reflecting the smaller volume of material intersecting the resonant slice. Despite the dramatically smaller signal, the signal-to-noise ratio is still quite respectable using $1 \mathrm{~min}$ of averaging per point. The contrast of the peaks is greatly helped by the low baseline noise level and minimal 


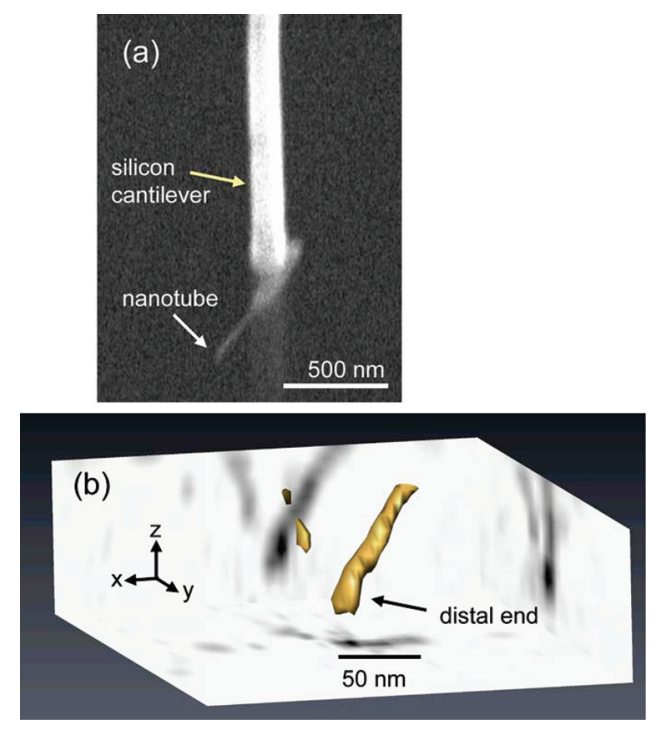

Figure 5. (a) Scanning electron micrograph of multiwalled carbon nanotube attached to a silicon cantilever (side view). The thin NT is supported by a thicker NT that was affixed to the cantilever and then thickened further via electron-beam deposited contamination. (b) Three-dimensional image reconstructed from a 3D MRFM data set. The rendered object represents a surface of constant hydrogen density.

background signal over the center of the tip, which are consequences of the restricted geometry of the nanotube.

A two-dimensional scan of the sharp nanotube shows a ringlike structure in Figure 4b. This ring gives a direct visualization of a cut through the paraboloidal PSF. The image is not purely a reflection of the PSF, however, because the object is not precisely a point object. Information about the object is contained within the image; for example, the asymmetry between the left and right lobe and the spatial extent of the lobes all reflect the detailed three-dimensional shape of the object. By taking a set of 2D scans at different tip-sample spacings, sufficient information can be obtained to determine the real space image of the object.

We used the same data collection scheme and Landweber deconvolution used previously in order to reconstruct a three-dimensional image. ${ }^{7,15,25}$ Four scan planes of data were taken with a grid spacing in $x$ and $y$ of $10 \mathrm{~nm}$ $\times 33 \mathrm{~nm}$ and a $z$-spacing of $21 \mathrm{~nm}$. The Landweber algorithm was used to iterate to a solution, taking roughly a thousand iterations. The result is shown as an isosurface (3D contour of constant density) in Figure 5b. The rendering shows a cylinder-like object, roughly $10 \mathrm{~nm}$ across at the distal end. Given that the data are quite spatially undersampled, the 3D image captures the geometry remarkably well, including the fact that the NT is cocked at a significant angle in the $x$ direction. The apparent roughness on the surface of the image is probably due to noise, as are the extraneous islands of material to the left of the nanotube. If the hydrogen giving rise to the signal is due exclusively to an adsorbed surface layer, one would expect the reconstructed image to be hollow. We have not seen any evidence for hollow structure in either the raw data or reconstructed image. Given that the effect of a hollow structure would be quite subtle in the data, it is likely that we do not have the sensitivity or resolution to detect it. It is also quite possible that the structure is in fact not hollow, but that hydrogen was incorporated into the structure either during initial growth of the nanotube or in subsequent mounting and exposure to the environment.

As an aside, we can analyze the NT data in the context of nanoscale magnetometry. There is currently a great deal of interest in such techniques, spurred in part by recent results in electron spin resonance of single nitrogen vacancy centers in diamond, which suggest that it is possible to combine nanoscale spatial resolution with field sensitivity in the nanotesla regime. ${ }^{26-28}$ Although MRFM is sensitive to magnetic moments and field gradients, not magnetic fields per se, it is nonetheless instructive to compute an effective field sensitivity. Using the field gradient from the tip model of $2.4 \times 10^{6} \mathrm{~T} / \mathrm{m}$ at tip-sample spacing $z=50 \mathrm{~nm}$ and the measured baseline noise level of $3.5 \mathrm{aN} / \mathrm{Hz}^{1 / 2}$ from the NT experiment, the corresponding demonstrated minimum detectable moment $\mu_{\min }$ is roughly $1.4 \times 10^{-24} \mathrm{~J} / \mathrm{T} \cdot \mathrm{Hz}^{1 / 2}$, or 100 spins-rms $/ \mathrm{Hz}^{1 / 2}$. One can associate this number with a minimum detectable field by determining the field that this moment would produce at a distance $z=50 \mathrm{~nm}$. The resulting minimum detectable field $B_{\min }=\mu_{0} \mu_{\min } / 2 \pi z^{3}=2.3$ $\mathrm{nT} / \mathrm{Hz}^{1 / 2}$ at $T=250 \mathrm{mK}$.

In summary, we have shown that MRFM can use the spectroscopic nature of magnetic resonance to give elemental selectivity in organic materials and have made use of the hydrogen selectivity to image thin films of natural adsorbates. The background signal from such adsorbed layers may present some difficulty for future MRFM imaging of molecular-scale objects based on hydrogen detection, perhaps necessitating in situ cleaning. At the same time, we have shown that the hydrogen can be used as a form of contrast agent to enable imaging of a multiwalled carbon nanotube. Nanotubes have some attractive properties, such as a spatially localized geometry that should reduce unwanted interactions with the sample and minimize any background signal. In the future, they might make an ideal platform for supporting samples such as viruses and macromolecules for MRFM, provided that the means of functionalization ${ }^{29}$ and attachment can be developed.

Acknowledgment. We thank B. Chui for the cantilever fabrication and M. Salmeron for helpful discussions. We acknowledge support from the NSF-funded Center for Probing the Nanoscale at Stanford University (NSF Grant PHY-0425897).

\section{References}

(1) Binnig, G.; Rohrer, H. Scanning tunneling microscopy. IBM J. Res. Dev. 1986, 30, 279-293.

(2) Stipe, B. C.; Rezaei, M. A.; Ho, W. Single-Molecule Vibrational Spectroscopy and Microscopy. Science 1998, 280, 1732-1735.

(3) Sugimoto, Y.; Pou, P.; Abe, M.; Jelinek, P.; Perez, R.; Morita, S.; Custance, O. Chemical identification of individual surface atoms by atomic force microscopy. Nature 2007, 446, 64-67.

(4) Stockle, R. M.; Suh, Y. D.; Deckert, V.; Zenobi, R. Nanoscale chemical analysis by tip-enhanced Raman spectroscopy. Chem. Phys. Lett. 2000, 318, 131-136.

(5) Sidles, J. A.; Garbini, J. L.; Bruland, K. J.; Rugar, D.; Zueger, O.; Hoen, S.; Yannoni, C. S. Magnetic resonance force microscopy. Rev. Mod. Phys. 1995, 67, 249-265. 
(6) Rugar, D.; Zuger, O.; Hoen, S.; Yannoni, C. S.; Vieth, H. M.; Kendrick, R. D. Force detection of nuclear magnetic resonance. Science 1994, 264, 1560-1563.

(7) Degen, C. L.; Poggio, M.; Mamin, H. J.; Rettner, C. T.; Rugar, D. Nanoscale magnetic resonance imaging. Proc. Nat. Acad. Sci. U.S.A. 2009, 106, 1313-1317.

(8) Kuehn, S.; Hickman, S. A.; Marohn, J. A. Advances in mechanical detection of magnetic resonance. J. Chem. Phys. 2008, 128, 052208.

(9) Poggio, M.; Rettner, C. T.; Degen, C. L.; Mamin, H. J.; Rugar, D. Nuclear magnetic resonance microscopy with a microwire rf source. Appl. Phys. Lett. 2007, 90, 263111.

(10) Sleator, T.; Hahn, E. L.; Hilbert, C.; Clarke, J. Nuclear-spin noise. Phys. Rev. Lett. 1985, 55 (17), 1742-1745.

(11) Mamin, H. J.; Budakian, R.; Chui, B. W.; Rugar, D. Magnetic resonance force microscopy of nuclear spins: Detection and manipulation of statistical polarization. Phys. Rev. B 2005, 72, 024413-1.

(12) Degen, C. L.; Poggio, M.; Mamin, H. J.; Rugar, D. Role of Spin Noise in the Detection of Nanoscale Ensembles of Nuclear Spins. Phys. Rev. Lett. 2007, 99, 250601.

(13) Crooker, S. A.; Rickel, D. G.; Balatsky, A. V.; Smith, D. L. Spectroscopy of spontaneous spin noise as a probe of spin dynamics and magnetic resonance. Nature 2004, 431, 49-52.

(14) Zueger, O.; Hoen, S. T.; Yannoni, C. S.; Rugar, D. Three-dimensional imaging with a nuclear magnetic resonance force microscope. J. Appl. Phys. 1996, 79, 1881-1884.

(15) Chao, S.-H.; Dougherty, W. M.; Garbini, J. L.; Sidles, J. A. Nanometerscale magnetic resonance imaging. Rev. Sci. Instrum. 2004, 75, 11751181.

(16) Verhagen, R.; Wittlin, A.; Hilbers, C. W.; Kempen, H. v.; Kentgens, A. P. M. Spatially resolved spectroscopy and structurally encoded imaging by Magnetic Resonance Force Microscopy of quadrupolar spin systems. J. Am. Chem. Soc. 2002, 124, 1588-1589.

(17) Thurber, K. R.; Harrell, L. E.; Smith, D. D. 170 nm nuclear magnetic resonance imaging using magnetic resonance force microscopy. $J$. Magn. Reson. 2003, 162, 336-340.

(18) Mitsui, T.; Rose, M. K.; Fomin, E.; Ogletree, D. F.; Salmeron, M. Water Diffusion and Clustering on Pd(111). Science 2002, 1850 1852 .
(19) Hu, J.; Xiao, X.-D.; Salmeron, M. Scanning polarization force microscopy: A technique for imaging liquids and weakly adsorbed layers. Appl. Phys. Lett. 1995, 67, 476-478.

(20) Chui, B. W.; Hishinuma, Y.; Budakian, R.; Mamin, H. J.; Kenny, T. W.; Rugar, D. Mass-loaded cantilevers with suppressed higherorder modes for magnetic resonance force microscopy. Technical digest 12th International Conference on Solid-State Sensors and Actuators (Transducers'03), Boston, 2003; IEEE: Piscataway, NJ, 2003; pp 1120-1123.

(21) Sowell, R. R.; Cuthrell, R. E.; Mattox, D. M.; Bland, R. D. Surface cleaning by ultrviolet radiation. J. Vac. Sci. Technol., B 1974, 11, 474475.

(22) Vig, J. R. UV/ozone cleaning of surfaces. J. Vac. Sci. Technol., A 1985, 3, 1027-1034.

(23) Mamin, H. J.; Poggio, M.; Degen, C. L.; Rugar, D. Nuclear magnetic resonance imaging with $90-\mathrm{nm}$ resolution. Nat. Nanotechnol. 2007, 2,301 .

(24) Colbert, D. T.; Zhang, J.; McClure, S. M.; Nikolaev, P.; Chen, Z.; Hafner, J. H.; Owens, D. W.; Kotula, P. G.; Carter, C. B.; Weaver, J. H.; Rinzler, A. G.; Smalley, R. E. Growth and sintering of fullerene nanotubes. Science 1994, 266, 1218-1222.

(25) Bertero, M.; Boccacci, P. Introduction to Inverse Problems in Imaging; Institute of Physics: Bristol, U.K., 1998; pp 137-167.

(26) Maze, J. R.; Stanwix, P. L.; Hodges, J. S.; Hong, S.; Taylor, J. M.; Cappellaro, P.; Jiang, L.; Dutt, M. V. G.; Togan, E.; Zibrov, A. S.; Yacoby, A.; Walsworth, R. L.; Lukin1, M. D. Nanoscale magnetic sensing with an individual electronic spin in diamond. Nature 2008, 455, 644-648.

(27) Balasubramanian, G.; Chan, I. Y.; Kolesov, R.; Al-Hmoud, M.; Tisler, J.; Shin, C.; Kim, C.; Wojcik, A.; Hemmer, P. R.; Krueger, A.; Hanke, T.; Leitenstorfer, A.; Bratschitsch, R.; Jelezko, F.; Wrachtrup, J. Nanoscale imaging magnetometry with diamond spins under ambient conditions. Nature 2008, 455, 648-651.

(28) Degen, C. L. Scanning magnetic field microscope with a diamond single-spin sensor. Appl. Phys. Lett. 2008, 92, 243111.

(29) Wong, S. S.; Joselevich, E.; Woolley, A. T.; Cheung, C. L.; Lieber, C. M. Covalently functionalized nanotubes as nanometre-sized probes in chemistry and biology. Nature 1998, 394, 52-55.

NL901466P 